Cahiers d'études africaines

\title{
La débâcle éducative du Kenya
}

Éléments d'analyse historique

\section{Hélène Charton}

\section{CpenEdition}

\section{Journals}

Édition électronique

URL : http://journals.openedition.org/etudesafricaines/196

DOI : 10.4000/etudesafricaines. 196

ISSN : 1777-5353

Éditeur

Éditions de l'EHESS

\section{Édition imprimée}

Date de publication : 1 janvier 2003

Pagination : 189-208

ISBN : 978-2-7132-1809-5

ISSN : 0008-0055

\section{Référence électronique}

Hélène Charton, "La débâcle éducative du Kenya », Cahiers d'études africaines [En ligne], 169-170 | 2003, mis en ligne le 20 décembre 2006, consulté le 19 avril 2019. URL : http:// journals.openedition.org/etudesafricaines/196 ; DOI : 10.4000/etudesafricaines.196 


\section{Hélène Charton}

\section{La débâcle éducative du Kenya

\author{
Éléments d'analyse historique
}

En mars 2000, 67 élèves de l'école secondaire de Kyanguli, près de Nairobi, étaient brûlés vifs dans leur dortoir. L'origine criminelle de l'incendie a révélé la violence qui ravage les établissements scolaires du Kenya ; le mauvais état des infrastructures scolaires a également contribué à alourdir le bilan humain de ce drame. Or cette tragédie, unique dans son ampleur, n'est cependant pas un phénomène isolé. Ces formes de violence qui se doublent depuis quelques mois de grèves scolaires répétées, accompagnent un phénomène inédit de déscolarisation. Le taux de scolarisation primaire du Kenya, qui fut longtemps le pays modèle en matière d'éducation, est passé de $95 \%$ en 1988 à $79 \%$ en 2000. La rapidité et l'extrême violence du phénomène nous invitent à nous interroger sur les facteurs historiques qui ont précipité le déclin de l'institution scolaire.

Au Kenya, la nature de la colonisation a stimulé le désir d'éducation de la population africaine de la colonie, frustrée par la domination de colons européens. Les initiatives populaires qui se sont alors développées ont été encouragées et institutionnalisées lors de l'indépendance en 1963. Mais le développement de l'éducation, qui reposait alors sur l'interaction dynamique entre les initiatives des communautés et l'aide du gouvernement, n'a pas résisté à la dégradation de la situation économique du pays. En dépit des tentatives de réformes, le système éducatif traverse depuis une décennie une crise sans précédent. Celle-ci scelle la rupture définitive entre deux logiques contradictoires que l'État affaibli n'est plus en mesure de conjuguer.

\section{Les paradoxes du développement des institutions scolaires}

La problématique scolaire entre héritage colonial et construction nationale

\section{Le legs colonial}

Le Kenya est une ancienne colonie de peuplement. Si le nombre des colons européens est resté relativement modéré, l'influence de cette communauté 
a été toutefois déterminante dans l'évolution sociale, économique et politique du territoire britannique ${ }^{1}$. Pour le bon développement de leurs exploitations agricoles, les colons ont eu besoin d'une main-d'œuvre efficace et docile que le gouvernement colonial s'est chargé de former dans les écoles de la colonie réservées aux jeunes Africains. L'éducation, qui leur était alors dispensée par les missionnaires, se limitait, en effet, aux apprentissages de base.

La marginalisation systématique de la population africaine au sein de la société coloniale a cependant suscité des mouvements de résistance. L'éducation académique réservée aux enfants des communautés immigrées a polarisé le désir de promotion sociale de la population africaine de la colonie. L'acquisition des mêmes diplômes que les Européens a rapidement été perçue par les Africains comme un moyen de se hisser au même rang social. C'est dans cette perspective que cette communauté a mis en place des initiatives éducatives originales à partir des années 1930 .

Les écoles indépendantes ont ainsi proposé en pays luo et kikuyu des modèles alternatifs d'éducation. Elles rejettent le monopole missionnaire et retiennent l'anglais comme langue d'enseignement au détriment des langues locales utilisées dans le système colonial. Les objectifs poursuivis par les écoles des assemblées locales sont identiques. Les Local Natives Councils $(\mathrm{LNC})^{2}$ ont constitué des fonds spéciaux pour financer des écoles secondaires - inexistantes au Kenya - et offrir des bourses d'études supérieures à l'étranger aux élèves les plus méritants ${ }^{3}$. Après la Seconde Guerre mondiale, les premières associations nationalistes du Kenya inscrivent les thématiques scolaires en tête de leurs revendications. En 1945, la Kenya African Union (KAU) adresse un mémorandum au Colonial Office, dans lequel elle demande que la scolarisation primaire universelle soit étendue aux jeunes Africains ${ }^{4}$.

Ces différentes initiatives témoignent de la place centrale de l'éducation pour les populations africaines de la colonie. L'École concentre, en effet, les espoirs de promotion sociale d'une communauté frustrée et marginalisée par l'environnement colonial. Le Kenya, devenu indépendant en décembre 1963, doit gérer ce profond désir de scolarisation.

1. En 1921, le Kenya compte 9600 Européens, pour une population totale estimée à 2,5 millions de personnes; c'est peu en comparaison des 50000 colons de la Rhodésie du Sud qui a alors une population similaire. Mais ce chiffre augmente considérablement après la guerre puisque le nombre des Européens présents au Kenya passe de 30000 à 50000 entre 1948 et 1962.

2. Les Local Native Councils sont établis en 1924 pour canaliser les premières revendications politiques des Africains.

3. En 1945, le Local Native Council du Nyanza consacre $30 \%$ de son budget au financement de projets éducatifs indépendants.

4. Cette mesure avait été adoptée pendant la guerre pour les deux communautés immigrées de la colonie, les Indiens et les Européens. Public Record Office, CO 533/557/1, Memorandum de la KAU. 


\section{L'école au service de l'édification de la nation}

Les écoles primaires de la jeune république du Kenya accueillent, à la rentrée de 1964, plus d'un million d'élèves, soit deux fois plus qu'en 1957 et quatre fois plus qu'en 1948 (Bogonko 1992 : 112). Cette explosion scolaire apparaît comme un phénomène de rattrapage après des années de frustration. Le nouveau gouvernement de la république du Kenya doit satisfaire le vif désir scolaire de la population tout en développant un programme d'éducation répondant aux besoins du jeune État. C'est autour de cette double contrainte - parfois paradoxale — que se développe le système scolaire après 1963.

Les responsables du pays s'efforcent alors de définir le rôle assigné au système éducatif dans le développement d'une nation indépendante. L'école doit diffuser et enraciner les valeurs de la société nouvelle tout en assurant l'intégration nationale et le développement du pays. Dans son Manifeste, en 1963, la Kenya African National Union (KANU), le parti politique dominant (et bientôt unique), désigne la scolarisation primaire universelle comme un objectif à atteindre en priorité, tandis que le premier ministre, Jomo Kenyatta, place l'éducation au centre de l'effort de développement demandé à la population du Kenya: "Nous devons travailler plus dur afin de combattre nos ennemis : l'ignorance, la maladie et la pauvreté. C'est pourquoi je lance cet appel : HARAMBEE! Travaillons dur et ensemble, pour notre pays, le Kenya »(Kenyatta 1965: 7) $)^{5}$.

L'expression harambee désigne moins l'effort que ses modalités. Cet appel à la mobilisation de toutes les énergies pour le développement du pays est un principe fondamental du projet de société, tel qu'il est défini dans le Sessional Paper $\mathrm{n}^{\circ} 10$ de 1965, intitulé African Socialism and its Application to Planning. L'expansion de la scolarisation primaire repose sur les efforts de la population tandis que le gouvernement se concentre sur les formations secondaires et supérieures. En effet, le Kenya indépendant manque, à tous les niveaux, de personnel qualifié et doit faire appel à des expatriés : «À ce stade du développement du Kenya, l'éducation est davantage un service économique que social et doit être considérée comme le principal moyen pour remédier aux pénuries de main-d'œuvre qualifiée et réduire les inégalités entre tous les citoyens $»^{6}$.

5. Discours du $1^{\text {er }}$ juin 1963. Texte original : «We must work harder to fight our enemies: ignorance, sickness and poverty. I therefore give you the call: HARAMBEE ! Let us all work hard together for our country, Kenya. »

6. Development Plan, Government of Kenya, Government Printer, 1965-1970. Le plan révisé en 1966 reprend les conclusions du rapport Ominde qui avait défini, en 1964, les orientations du système éducatif du Kenya dans le cadre de l'indépendance. Texte original : "At this stage of Kenya's development, education is much more an economic than a social service and should be regarded as the principal means for relieving the shortage of skilled manpower and equalizing economic opportunities among all citizens. » 
Le nouveau gouvernement insiste donc sur la nécessité de doter le Kenya d'un système éducatif qui soit adapté à ses réalités sociales et économiques : «Une éducation complètement tournée vers les emplois urbains de cols blancs ne peut que désorienter, décevoir et frustrer la majorité des élèves qui quittent l'enseignement primaire car c'est dans l'agriculture que la majorité des Kenyans pourra être employée ${ }^{7}$.

Toutefois, aucune réforme des programmes n'est réalisée. Les enseignements traditionnels continuent d'être privilégiés au détriment de matières plus pratiques comme l'agriculture. Cela traduit l'ambiguïté du développement de l'éducation au Kenya après 1963. L'École doit satisfaire les besoins de la jeune république en formant le personnel qualifié dont elle a besoin. Mais l'éducation doit aussi répondre au désir de la population à qui est confiée une part importante de son développement. L'équation des besoins et du désir se révèle délicate à résoudre ; les modalités d'expansion du système éducatif du Kenya permettent d'apprécier la relation parfois contradictoire qu'entretiennent ces deux logiques.

\section{Les modalités et les enjeux de l'expansion scolaire}

\section{La philosophie harambee appliquée au développement scolaire}

Les initiatives gouvernementales et les efforts de la population se répondent dans une relation dynamique. L'État prend le contrôle des écoles primaires et tous les instituteurs deviennent des fonctionnaires. Les programmes sont unifiés dans le cadre du Primary Syllabus de 1967. De leur côté, les populations satisfont leur soif d'éducation en construisant partout de nouvelles écoles (Bogonko 1992 : 118). Ce mouvement, d'abord spontané, est rapidement intégré au processus d'expansion du système éducatif, dans le cadre de la philosophie harambee. Dès lors, il est acquis que les communautés prennent en charge le développement des bâtiments et des équipements scolaires. Les parents contribuent également à la scolarisation de leurs enfants en s'acquittant de frais de scolarité qui représentent en moyenne, en 1970, $33 \%$ des budgets de fonctionnement des écoles ${ }^{8}$.

Ces efforts sont régulièrement récompensés par le gouvernement qui prend des mesures spectaculaires. En décembre 1973, pour célébrer les dix années d'indépendance et de pouvoir, le président Jomo Kenyatta abolit les frais de scolarité pour les quatre premiers niveaux du primaire. Les frais

7. Development plan, Government of Kenya, Government Printer, 1965-1970. Texte original: "An education that places unwarranted emphasis on "urban" white collar employment can only mislead, disappoint and frustrate the majority of primary school leavers since it is in agriculture that the majority of Kenyans will find their employment. »

8. Development Plan, Government of Kenya, Government Printer, 1970-1974, p. 546. 
de scolarité sont, dans les trois autres niveaux standardisés, de 60 shillings kenyans ${ }^{9}$. L'effet de cette mesure est immédiat, comme le souligne le graphique suivant :

GRAPHiQUE I. — SCOLARISATION PRIMAIRE PAR NIVEAU ENTRE 1965 ET 1976

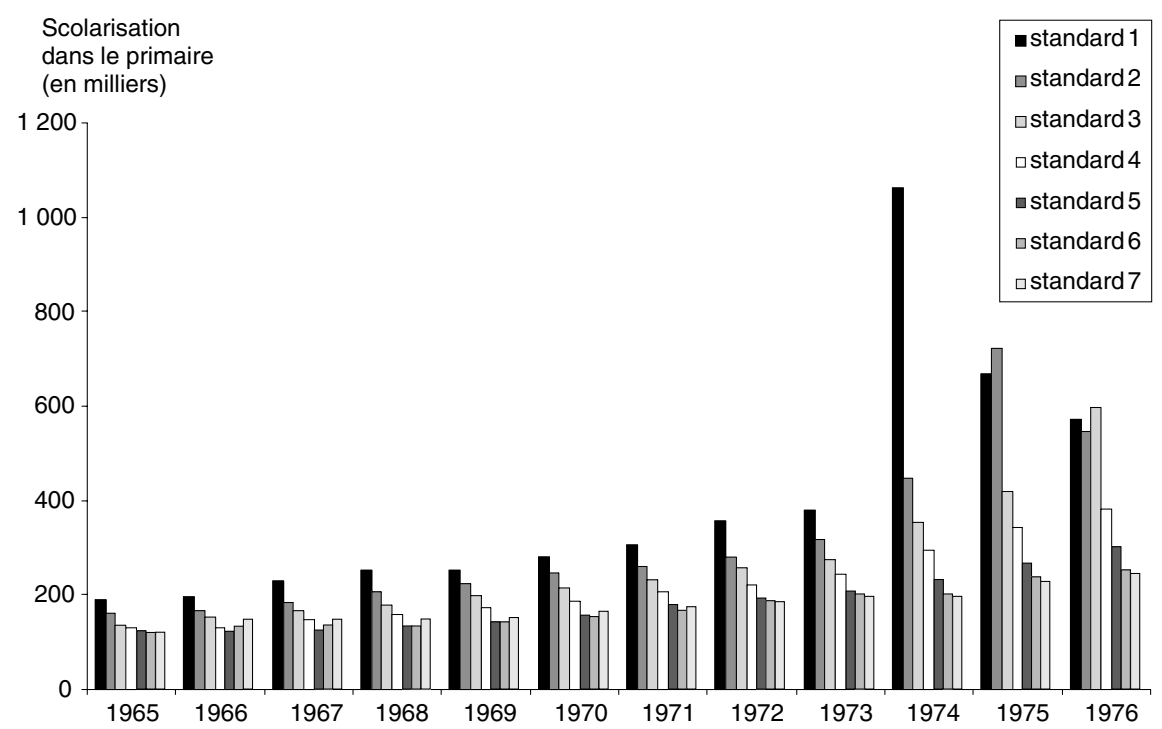

Source : Government of Kenya, Statistical Abstracts, 1966-1995, Nairobi.

En janvier 1974, les écoles primaires accueillent $49 \%$ d'élèves en plus (la progression est de $53 \%$ pour les filles), alors que la croissance était de $9 \%$ les deux années précédentes. Mais le graphique montre également les limites du décret présidentiel de 1973. Le gonflement de 1974 est essentiellement constitué d'élèves de la première classe. Mais surtout, ce recrutement exceptionnel ne se maintient pas et décroît au contraire très rapidement dans les années suivantes, car en réalité la gratuité de l'École n'est qu'apparente. La demande scolaire, très forte en 1974 (parfois même anticipée par les parents), nécessite de construire rapidement de nouvelles classes, ce qui entraîne l'apparition de nouveaux droits dont le montant total dépasse souvent les frais de scolarité demandés avant la gratuité. C'est sans doute pour cette raison que l'effectif scolaire n'atteint pas les prévisions du plan de développement de 1974-1978, qui annonçaient plus de trois millions d'élèves pour 1978.

En 1979, le successeur du président-fondateur, Daniel Arap Moi, inaugure son mandat en étendant la gratuité de l'École à toutes les classes du

9. Development Plan, Government of Kenya, Government Printer, 1974-1978, p. 410. 
primaire. Le gouvernement abolit notamment les droits supplémentaires exigés dans les écoles primaires au titre de la participation des parents aux fonds de construction, activités extra-scolaires et autres, qui biaisaient la gratuité de principe. En complétant certaines mesures de 1974, le nouveau président inscrit résolument son action dans la continuité de celle de son prédécesseur et fait un geste décisif en faveur de la population (Bogonko 1992 : 115). Les effets de cette nouvelle mesure sont plus durables, comme le montre le graphique suivant :

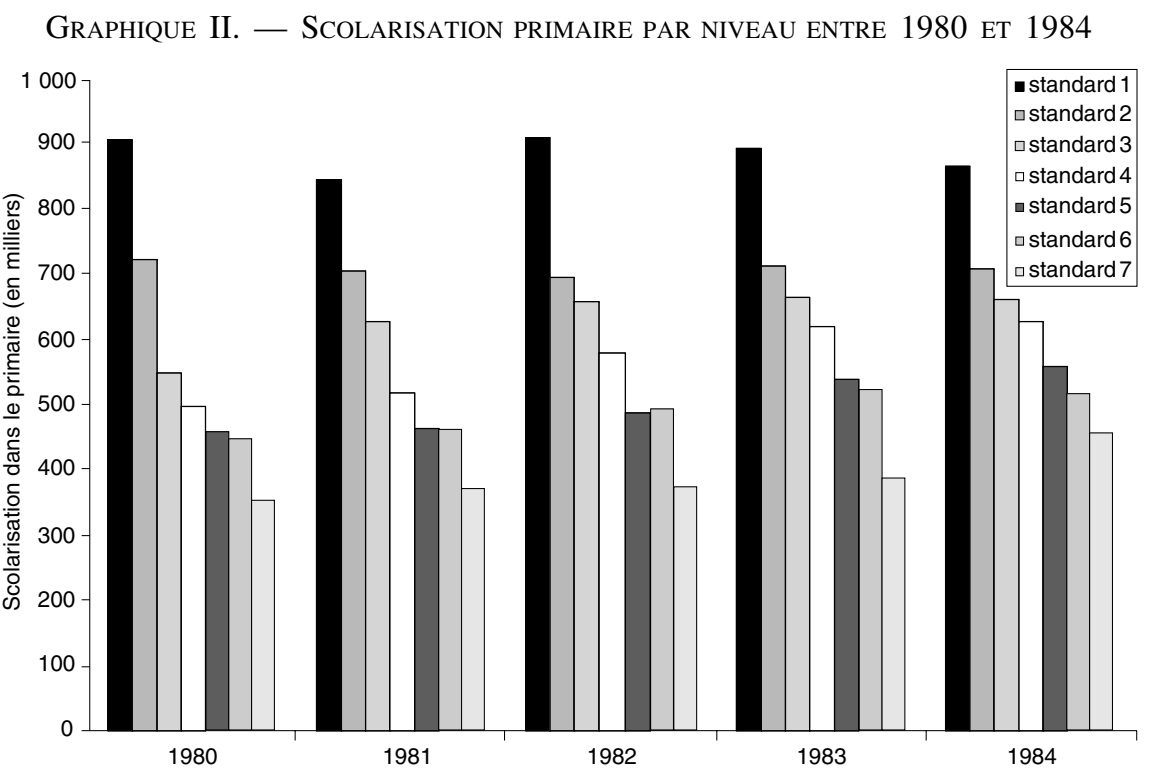

Source: Statistical Abstracts, op. cit.

En effet, même si la déperdition reste forte entre les différentes classes, le recrutement en standard 1 (la première classe) se maintient à un niveau élevé. Ces mesures exceptionnelles ont des répercussions sur l'ensemble du système scolaire du Kenya, comme on peut le lire sur le graphique qui présente l'évolution de la scolarisation primaire et secondaire entre 1963 et 1984.

Les décrets présidentiels de 1973 et 1979 apparaissent nettement dans ce graphique ; on peut également observer leurs répercussions au niveau du secondaire. C'est, proportionnellement, le second cycle qui a le plus bénéficié de l'expansion du système scolaire après l'indépendance. Alors que le nombre des élèves des écoles primaires est passé de 1 à 3 millions entre 1963 et 1978, celui du secondaire à été multiplié par 12. La scolarisation primaire «pour tous » a généré une forte demande au niveau du secondaire que les écoles du pays ne sont pas en mesure d'absorber. Par conséquent, 
GraPHIQUE III. - Évolution DE LA SCOLARISATION PRIMAIRE ET SECONDAIRE ENTRE 1963 ET 1984

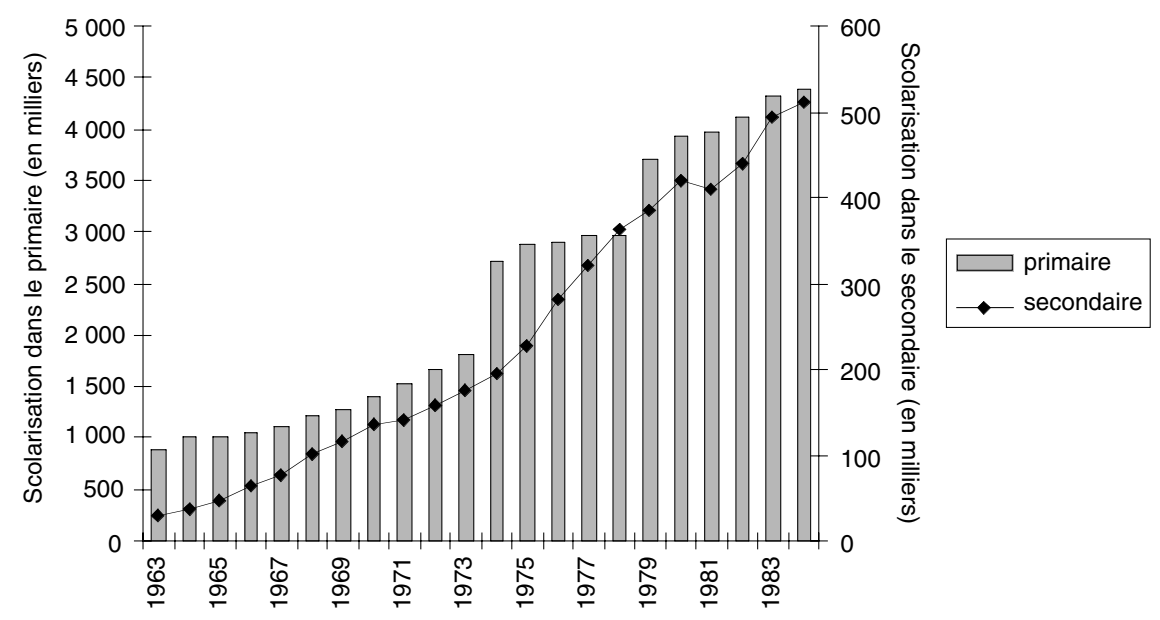

Source: Statistical Abstracts, op. cit.

le passage du premier au second cycle est devenu plus sélectif, renforçant ainsi le caractère élitiste du système scolaire du Kenya.

\section{Les stratégies éducatives de la population du Kenya}

Pour être admis dans le secondaire, les élèves du primaire doivent réussir le Certificate of Primary Education (CPE). Ceux qui obtiennent les meilleurs résultats à cet examen se disputent les quelques places disponibles dans les écoles gouvernementales qui sont à la fois les plus performantes et les moins chères.

Depuis 1965, les candidats au CPE sont évalués sur la base de questionnaires à choix multiples. L'enjeu important que représente la réussite de cet examen a rapidement transformé les derniers niveaux du primaire en classes de bachotage. Les élèves s'efforcent de mémoriser les annales, et la maîtrise de cette technique de l'examen ne laisse aucune place à l'initiative pédagogique (Sifuna 1980). Les résultats de l'enquête de terrain réalisée par Lewinger Moock, en 1970, auprès de 139 élèves de standard 7 du district de Maragoli, zone rurale de l'Ouest du Kenya, permettent d'apprécier les enjeux du CPE. Seuls $5 \%$ des candidats entrent dans une école gouvernementale; ils sont en revanche $19 \%$ à trouver une place dans les écoles privées ; enfin, $50 \%$ préfèrent redoubler cette dernière classe, dans l'espoir de faire mieux l'année suivante. Certains élèves n'hésitent pas à tricher sur leur âge, ou même leur nom, pour pouvoir se réinscrire (Lewinger Moock 1973). L'expérience de Maragoli est révélatrice de la situation générale du 
Kenya. En 1968, seuls $8 \%$ des candidats au CPE ont été admis dans une école secondaire gouvernementale ${ }^{10}$, contre $13 \%$ en $1975^{11}$. Or, sur la même période, la proportion des élèves poursuivant des études secondaires, toutes écoles confondues, est passée de moins de $20 \%$ à plus de $30 \%$. Cette augmentation profite donc aux écoles privées et communautaires qui connaissent alors un fort développement.

Afin de conjurer la forte sélection à l'entrée dans le secondaire et permettre au plus grand nombre possible de poursuivre leurs études, les communautés ont mis en place un réseau d'écoles auto-financées, appelées écoles harambee. Leur proportion a évolué de la façon suivante :

Graphique IV. - Proportion DES ÉCOLES SECONDAIRES HARAMBEE

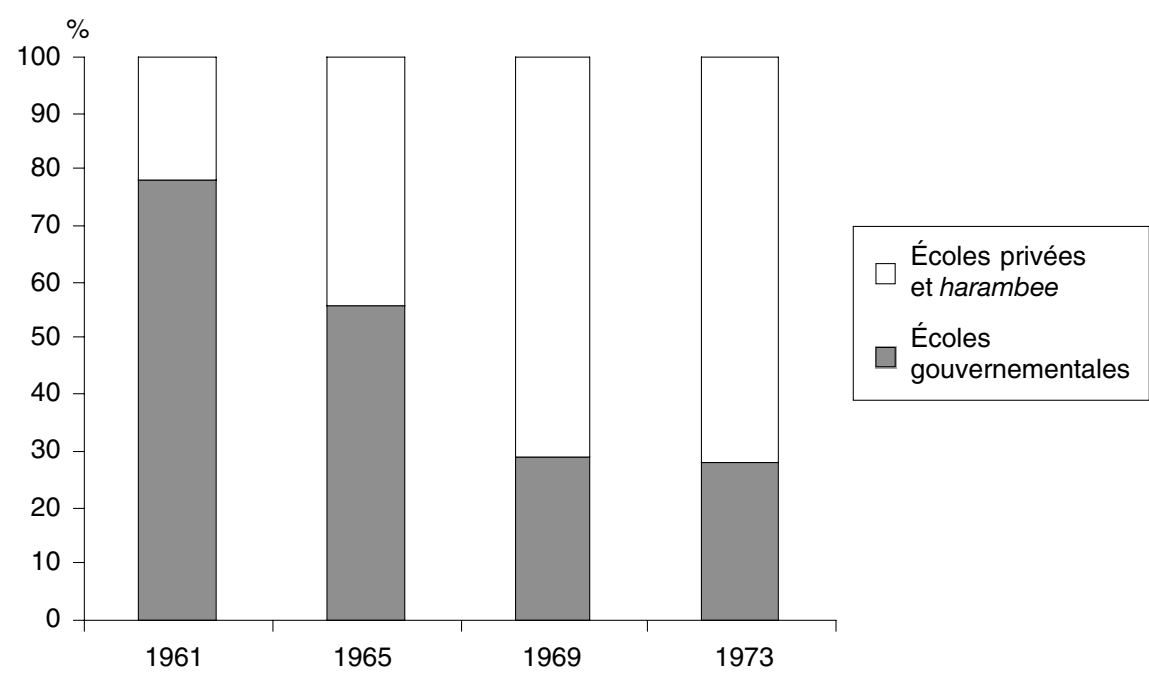

Source: H. Charton, Le désir d'école, Paris, Publications de l'Université Paris 7, 1997, p. 49.

Le nombre de ces écoles dépasse rapidement celui des établissements gouvernementaux. Ces lycées coûtent pourtant cher et leurs résultats aux examens nationaux sont médiocres; ils souffrent de la faiblesse de leurs moyens (enseignants peu qualifiés, équipements limités). Mais ces initiatives témoignent de la vivacité du désir d'éducation de la population auquel le gouvernement répond en intégrant progressivement les écoles harambee au système public. Dès qu'elles ont atteint un niveau jugé suffisant, l'État leur accorde des subventions ; en 1985, la moitié des écoles secondaires du Kenya avait ainsi une origine harambee. Mais le nombre sans cesse croissant des élèves achevant un cursus secondaire dévalorise ces diplômes, et

10. Development Plan, Government of Kenya, Government Printer, 1970-1974, p. 485.

11. Gachathi Report, Government of Kenya, Report of the National Committee on Educational Objectives and Policies, Nairobi, Government Printer, 1976, p. 49. 
la demande scolaire se reporte sur le niveau supérieur. Le recrutement de l'université devient alors plus sélectif et exclut les élèves des harambee d'un niveau plus faible que leurs collègues des lycées publics. Ainsi, en 1970, $48 \%$ des candidats entraient à l'université mais ce taux tombe à $23 \%$ en 1979 (Eshiwani 1993 : 72). Les étudiants cherchent alors des solutions alternatives qui reposent aussi sur des financements privés.

Au total, le système éducatif du Kenya s'est développé grâce à l'interaction des initiatives des populations et de l'action du gouvernement. Ce mode original d'expansion témoigne de la place centrale occupée par l'éducation dans la société kenyane. Mais cette dynamique trouve bientôt ses limites.

L'effort harambee demandé à l'indépendance a placé l'initiative populaire au cœur du développement de la scolarisation. Il apparaît donc logique que l'expansion scolaire ait obéi à des motivations essentiellement individuelles mais qui sont en contradiction avec la vocation sociale et intégrative de l'École définie à l'indépendance. Le gouvernement encourage et cautionne des initiatives communautaires qui favorisent paradoxalement une culture privée de l'éducation conçue (étant donnée la forte mobilisation financière qu'elle implique) comme un investissement productif. Or, plus l'investissement est lourd et plus il devient impératif de le rentabiliser. L'équilibre est donc fragile entre la valeur sociale et l'apport individuel qui sous-tend ce système. Il est menacé lorsque le diplôme ne garantit plus la mobilité sociale escomptée. La demande scolaire excédant l'offre, l'École devient un lieu de vive concurrence individuelle génératrice de frustration.

\section{Le système éducatif du Kenya en faillite}

Le financement d'un système éducatif en expansion continue n'est possible que dans un climat de relative prospérité économique. Mais la situation économique se dégrade rapidement au début des années 1980. La réforme des institutions scolaires qui est alors envisagée se fait sur fond de libéralisme économique. Le désengagement de la puissance publique dans le domaine de l'éducation est alors vécu par les populations toujours très sollicitées comme une trahison. La frustration est grande et le malaise social commence alors à envahir la scène scolaire.

\section{L'impossible réforme du système éducatif}

En dépit de la contribution active de la population kenyane, la croissance de la population scolaire pèse lourdement sur le budget du pays. Le salaire des enseignants, qui absorbe $90 \%$ du budget de l'enseignement primaire, est notamment un poste en perpétuelle expansion. La part du budget de 
l'État consacrée à l'éducation est ainsi passée de 11,9\% à 24,7 \% entre 1963 et 1974 . En 1985 , ce poste représentait toujours $18,1 \%$ du budget et $6,4 \%$ du PNB (Eshiwani 1993: 134).

\section{Un contexte économique défavorable}

Les mesures spectaculaires de 1974 et 1979 coïncident avec le retournement de la conjoncture économique du pays. Après une décennie de croissance, la situation se dégrade après 1973 comme le montre le tableau suivant:

\begin{tabular}{c|c}
\hline Dates & $\begin{array}{c}\text { Croissance annuelle } \\
\text { du PIB }\end{array}$ \\
\hline $1964-1972$ & $6,5 \%$ \\
$1973-1979$ & $4,7 \%$ \\
$1980-1987$ & $3,8 \%$ \\
\hline
\end{tabular}

Sources : B. A. Ogot, Decolonisation and Independence in Kenya, Nairobi, East African Studies, 1995, pp. 151-153.

Le gouvernement adopte un premier plan d'austérité pour 1974-1978 mais qui ne semble pas endiguer la récession puisque le PNB par habitant passe de 420 à 330 dollars US entre 1980 et 1987 (Ogot 1995 : 151). Le chômage des diplômés est l'une des manifestations de cette crise. Entre 1970 et 1983, les lauréats de l'Université de Nairobi passent de une à trois années à la recherche d'un emploi ; ils sont également nombreux à accepter un travail temporaire, tandis que beaucoup n'utilisent pas leur formation académique dans leur vie professionnelle (Banque Mondiale 1988 : 71). Le développement des formations supérieures et secondaires depuis 1963, renforcé par les initiatives harambee, a produit un nombre important de diplômés que le marché du travail saturé n'est pas en mesure d'absorber.

Il est alors nécessaire et urgent d'ajuster les filières de formation au nouveau contexte économique du pays. En 1975, le National Committee on Educational Objectives and Policies (NCEOP, plus connu sous le nom de son rapporteur, Gachathi) est chargé de définir les nouveaux buts de l'enseignement. Il s'agit notamment d'adapter le développement scolaire aux ressources et aux besoins du pays : "Le système actuel se caractérise par une très forte sélectivité. Son objectif est de produire un petit nombre d'individus capables de s'intégrer au secteur formel et moderne de l'économie. Sa nature très sélective et son orientation exclusive vers le secteur moderne et urbain sont fondamentalement en contradiction avec les valeurs sociales et culturelles défendues par le gouvernement $»^{12}$.

12. Development Plan, Government of Kenya, Government Printer, 1974-1978, p. 404. Texte original : «The present system is highly selective in character. Its objective is to product a few individuals who are equipped for placement in the modern formal sector of the economy. Its highly selective nature and exclusive orientation towards the modern urban sector are in fundamental contradiction in the social and cultural values upheld by the Government. » 
Le rapport propose une refonte globale du système scolaire dans la perspective d'une plus grande intégration à la vie économique du pays. L'enjeu principal de ses conclusions est de remédier au chômage des diplômés tout en brisant l'association, toujours persistante dans l'opinion, entre diplôme académique et travail salarié, devenue un mythe dans le contexte économique du pays. Mais la participation active de la population à l'effort de scolarisation est précisément stimulée par la perspective d'un travail salarié. Une telle réforme a donc des implications sociales et politiques majeures.

\section{La réforme en question}

La réforme scolaire du Kenya, élaborée depuis 1981 et entrée en application en 1985, s'inscrit donc dans un contexte économique de récession. Sous la pression de ses bailleurs de fond, le Kenya s'engage au début des années 1980 dans un programme de réformes économiques. Les contraintes d'allégement budgétaire génèrent des coupes dans des domaines qui ne sont pas jugés strictement nécessaires à la survie de l'État, et notamment l'éducation. L'enseignement est un investissement productif lourd pour l'État qui doit en retour servir le développement. Or, dans un contexte de rigueur économique, l'effort du gouvernement se concentre sur les besoins les plus urgents, en l'occurrence l'enseignement primaire, moins coûteux. En effet, en 1985, alors que $60 \%$ du budget de l'éducation va à l'enseignement primaire contre $12 \%$ pour le supérieur, la formation d'un étudiant permettrait la scolarisation de 60 écoliers (Banque Mondiale 1988 : 76). La réforme porte la durée du premier cycle d'enseignement à huit années au lieu de sept auparavant et pour les cycles secondaires et universitaires à quatre ans chacun (au lieu de six et trois ans) ; ce sont les étudiants qui financent leurs études. L'État se charge toutefois d'élargir les opportunités d'éducation supérieure. Trois nouvelles universités publiques sont créées et l'University Act de 1985 met en place la Commission on Higher Education (CHE) qui autorise ou non l'habilitation des universités privées (Eshiwani 1993: 68).

Cette réforme est assortie d'une modification des programmes qui privilégient, à tous les niveaux, les disciplines techniques et agricoles afin de rendre les enseignements plus adaptés aux besoins du pays. L' « agriculture » est ainsi pour la première fois réintroduite dans les écoles du Kenya. Enfin, la cohérence interne de chaque cycle d'enseignement, pensé comme une entité indépendante, est renforcée ; pour lutter contre les abandons, forme de gaspillage scolaire, un système de passerelles permet d'intégrer, dans des filières spécialisées, les élèves qui quittent le cursus académique en cours de scolarité ${ }^{13}$.

13. Ministry of Education, Government of Kenya, Nairobi, Government Printer, 1984, pp. 3-8. 
Les nouvelles orientations du système éducatif, diamétralement opposées à celles qui avaient été définies en 1963, répondent à un souci d'efficacité et obéissent à une logique libérale. Le désengagement de l'État au profit de l'initiative privée est alors officialisé. Le principe de la participation des bénéficiaires au financement des services sociaux appliqué à l'éducation secondaire et supérieure en est une illustration. Mais dans ce nouveau contexte économique, la mise en œuvre de la réforme repose sur la participation active de la population du Kenya.

\section{Coût et financement de l'éducation}

L'allongement d'un an de la durée du cycle primaire nécessite la construction de nouvelles salles de classes. Les communautés se mobilisent en organisant des collectes de fonds harambee. L'effort demandé est d'autant plus important que les nouveaux programmes exigent des équipements spéciaux (laboratoires, ateliers, etc.). En janvier 1985, 13370 classes accueillent les 46273 nouveaux élèves de standard 8. Mais bien souvent ces classes ne sont que des pièces vides. Leur équipement donne alors lieu à de nouvelles collectes de fonds. Ainsi l'école Old Kihumbuini, à Nairobi, demande 125 shillings à chaque parent d'élève pour acheter le mobilier des nouvelles classes (ils avaient déjà versé 500 sh chacun pour leur construction $)^{14}$. Ce mode de financement renforce les inégalités régionales puisque la réalité de la réforme dépend de la capacité de financement de chaque région.

Depuis 1985 , le coût de l'École n'a cessé d'augmenter ${ }^{15}$. Les participations extraordinaires semblent désormais institutionnalisées; les collectes de fonds harambee ont pris un caractère obligatoire et le moindre service assuré par l'institution scolaire prend la forme d'une participation supplémentaire. Tandis que le paiement des frais de scolarité est une angoisse réelle, partagée par une part importante de la population, la thématique scolaire est détournée à des fins politiques.

Les collectes de fonds harambee sont devenues des plates-formes politiques pour les élites locales. Les contributions de tel ministre ou député servent finalement moins le projet proposé que la promotion politique de ces personnalités. En janvier 1985 par exemple, une collecte harambee, organisée pour la construction d'écoles primaires à Bomet, réunit dans son fief le président Moi, qui fait un don personnel de 122000 shillings, et de nombreux ministres qui apportent également chacun leur contribution au projet pour un montant final de $2167543 \mathrm{sh}^{16}$. L'initiative échappe alors

14. Daily Nation, Nairobi, 9 janvier 1985.

15. La scolarisation d'un enfant coûte aujourd'hui en moyenne 10000 sh par an.

16. Daily Nation, Nairobi, 15 janvier 1985. 
aux communautés, contraintes de participer à des actions dont les réalisations sont de plus en plus hypothétiques. Le coût de l'École est également devenu une thématique électorale privilégiée. Le président annonce régulièrement l'abolition des contributions extraordinaires, vite rétablies par les School Committees. Ces fausses mesures contribuent à exaspérer l'opinion publique $^{17}$.

Tout considéré, le nouveau contexte économique qui encourage le désengagement de l'État modifie en profondeur les équilibres qui avaient présidé à la mise en place et au développement du système scolaire du Kenya depuis l'indépendance. La rigueur budgétaire limite l'investissement de l'État dans le domaine éducatif. Les communautés et les individus toujours fortement sollicités ne sont plus « récompensés » par des mesures spectacles comme les décrets de 1974 et 1979. L'amertume est d'autant plus vive que la réforme en question ne répond pas vraiment à leurs attentes. La relation dynamique entre l'initiative privée et le geste public, fondement de la philosophie harambee, ne fonctionne plus. La nature des collectes de fonds harambee illustre le détournement des thématiques scolaires à des fins politiques et électorales. Dans ces conditions, l'éducation ne fédère plus la nation dans un effort commun, mais est source d'inégalités nouvelles. La fréquentation des établissements scolaires a alors tendance à reculer et les violences qui se multiplient sont les révélateurs d'une crise inédite.

\section{Des systèmes de formation discrédités}

\section{L'École publique désavouée}

Depuis le début des années 1990, l'institution scolaire du Kenya a perdu de son prestige. Le phénomène de déscolarisation, qui touche pour la première fois le pays, traduit la perte de confiance de la population dans son système de formation. Le graphique $\mathrm{V}$ montre l'évolution de la scolarisation entre 1985 et 1995 .

Après une phase de croissance jusqu'en 1989, la scolarisation primaire stagne dans les années suivantes (elle chute en 1993), tandis que la scolarisation secondaire recule nettement après 1989. Cette désaffection scolaire qui touche les deux cycles d'études de manière cohérente a des causes diverses. Le coût de l'École est devenu souvent prohibitif. Or, un enfant qui n'a pas payé les frais de scolarité en temps voulu ou qui se présente sans uniforme est renvoyé chez lui (Mbeo Manyasi 2000). De plus, certains parents ne voient plus l'intérêt d'investir dans une éducation aux débouchés hypothétiques. Le nombre de places disponibles dans les écoles secondaires se réduit

17. Daily Nation, Nairobi, 3 février 1997. 
GRAPHIQUE V. - ÉvOlUTION DE LA SCOLARISATION PRIMAIRE ET SECONDAIRE ENTRE 1985 ET 1995

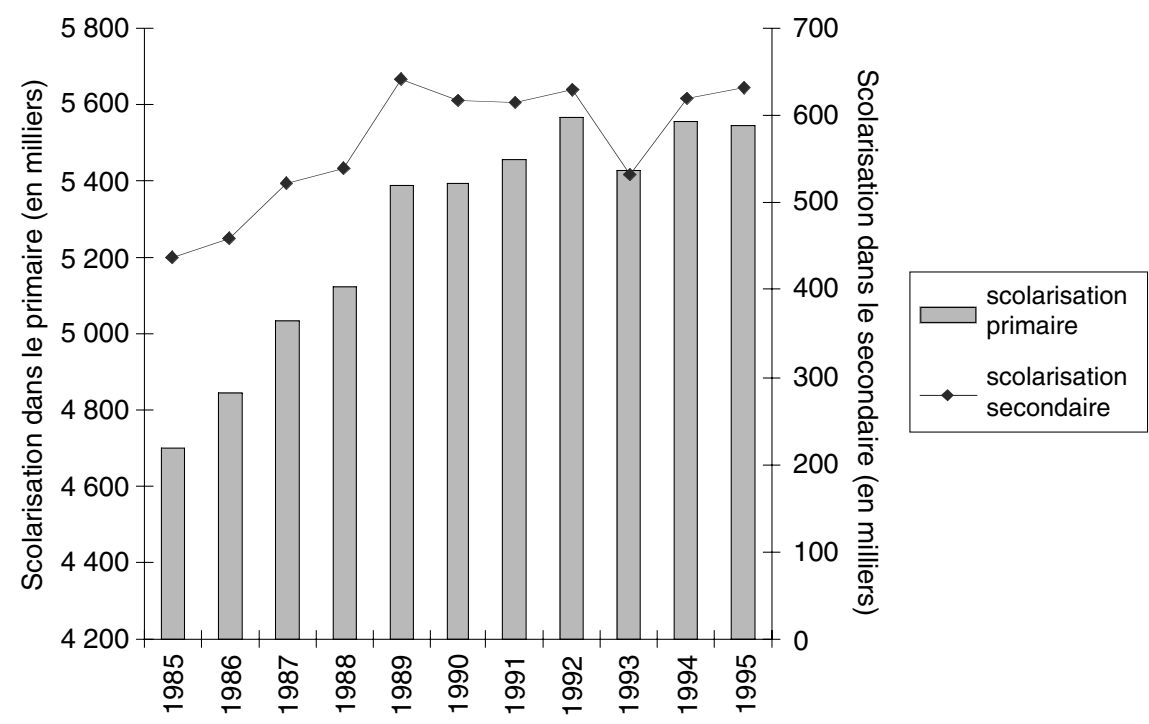

Source: Statistical Abstracts, op. cit.

chaque année. Le tableau ci-dessous souligne, en effet, la forte sélectivité du système scolaire.

\begin{tabular}{c|c}
\hline & \multicolumn{2}{|c}{ Élèves admis } \\
Dates & dans le secondaire (\%) \\
\hline 1992 & 49 \\
1994 & 43 \\
1995 & 46 \\
1997 & 44 \\
\hline
\end{tabular}

Sources: Kenya Statistical Abstracts, op. cit. et Daily Nation, Nairobi.

La nécessité de rentabiliser l'investissement scolaire détermine alors de nouvelles polarisations. Pour répondre à cette vive concurrence, des écoles privées, garantissant la réussite de leurs élèves au $\mathrm{KCPE}^{18}$, commencent à apparaître. En 1990, le Kenya compte 290 écoles primaires privées, généralement situées en milieu urbain. À la différence des écoles harambee, il s'agit d'initiatives strictement individuelles et commerciales et non plus communautaires. Représentant $1 \%$ seulement des élèves, les candidats de ces écoles ont pris les 10 meilleures places et la moitié des 50 premières

18. Le Kenya Certificate of Primary Education (KCPE) remplace depuis 1985, le CPE. 
au KCPE de $1996^{19}$. Les listes d'attente de ces écoles s'allongent, tandis que les prises à parti des enseignants à propos des mauvais résultats obtenus au KCPE continuent de se multiplier dans les écoles publiques ${ }^{20}$. Le grand succès de ces écoles privées révèle les failles du système public d'enseignement. En scolarisant leurs enfants dans ce type d'établissement, les parents tentent de leur donner une meilleure chance d'accéder à l'éducation secondaire et supérieure.

Mais le gouvernement s'inquiète du développement de ces écoles qui, selon lui, mettent en péril sa politique «d'uniformisation» de l'accès à l'éducation. Les menaces régulièrement proférées contre ces établissements relèvent davantage du discours électoral. Au nom de l'égalité des chances, chaque région dispose d'un quota de places dans les écoles secondaires, mais les critères de leur répartition ne sont pas communiqués. De nombreuses voix s'élèvent contre un système qui, sous couvert d'égalité, tendrait plutôt à favoriser l'assise électorale du président.

\section{La faillite des écoles du Kenya}

C'est pourtant ce souci avoué d'égalité qui a conduit le gouvernement à limiter le montant des frais de scolarité dans les écoles ${ }^{21}$ et à interdire les contributions supplémentaires. Bien que le gouvernement soit resté ferme face aux protestations des directeurs d'écoles qui jugent ces mesures irréalistes, ces dernières ont, semble-t-il, précipité le déclin des institutions scolaires du pays en entraînant d'importants problèmes de gestion ${ }^{22}$.

Le poids des matières pratiques et techniques dans les programmes génère des dépenses importantes. Le Kenya National Examination Council demande en effet chaque année aux établissements d'acheter du matériel neuf. Les laboratoires sont, par exemple, toujours très bien équipés même s'ils restent inutilisés faute de personnel qualifié. Pour boucler leur budget, les chefs d'établissements ont alors recours à divers expédients. Certains rognent sur la nourriture et les services offerts, tandis que d'autres préfèrent gonfler leurs effectifs pour accroître leurs revenus. Une inspection menée en 2001 dans 52 écoles secondaires du pays confirme la situation pathétique de ces établissements. Les dortoirs sont surpeuplés et, les réfectoires étant souvent inexistants, les étudiants mangent dans les salles de classe ou sous les arbres. Mais l'état lamentable des équipements de base coexiste souvent avec des installations luxueuses. Les chefs d'établissements préfèrent, en

19. Daily Nation, Nairobi, 3 janvier 1997.

20. The East African Standard, Nairobi, 21 janvier 1996.

21. Ils ne doivent pas dépasser 28900 sh dans les écoles nationales, 22900 sh dans les écoles provinciales et $10500 \mathrm{sh}$ dans les externats.

22. Daily Nation, Nairobi, 6 août 2001. 
effet, financer des projets prestigieux plutôt que remettre en état les équipements de base. Celui-ci achète deux bus de luxe alors que les dortoirs tombent en ruine et celui-là se lance dans la construction de nouveaux bureaux pour un montant de trois millions de shillings, alors que 15 étudiants se partagent un livre (Aduda 2001).

Certaines de ces pratiques renforcent le discrédit des écoles publiques : l'augmentation des frais de scolarité exclut une partie de la population, et la corruption qui permet aux parents de contourner la forte sélectivité des écoles nationales et aux établissements de se renflouer se répand. Des écoles reconnues accueillent alors de riches élèves faibles et démotivés. Dès lors, le fossé qui s'est creusé au sein de la société kenyane se projette sur les établissements scolaires. La presse rapporte les difficultés nouvelles auxquelles sont confrontées les écoles : «Les week-ends de sortie et les journées de visite sont devenus de véritables parades au cours desquelles les riches exhibent leurs richesses, tandis que les pauvres se tiennent en retrait plein de regrets. Les riches parents apportent à leurs enfants toutes sortes de produits chers et agréables de telle sorte qu'ils n'ont pas besoin de manger au réfectoire. Lorsque la nourriture de l'école est réservée aux pauvres, cette polarisation affecte durement leur moral $»^{23}$.

Dans ces conditions, l'éducation n'est plus un thème consensuel mais une source de conflits. La frustration qu'elle génère se manifeste alors à travers des formes de violence inédites.

\section{L'École dans l'impasse}

La multiplication des grèves et des violences dans les écoles au cours des deux dernières années illustre la faillite du système éducatif du pays et l'exaspération de la population. En juillet 2001, une cinquantaine d'écoles du pays se met en grève ${ }^{24}$. Le mouvement, qui est parti des écoles provinciales, s'étend aux établissements nationaux les plus prestigieux comme l'Alliance Girls High School, qui n'avait jamais connu de grève depuis sa fondation en 1948. Les revendications des jeunes grévistes portent sur les conditions de vie dans les écoles et notamment sur la nourriture et les sorties.

23. Daily Nation, Nairobi, 30 juillet 2001. Texte original : «The weekend outings and visiting days had been turned into exhibition parades where the rich flaunt their wealth and the poor sit back regretfully. The rich parents bring all sort of expensive niceties for their children so that they don't find the need to eat from the dining hall. And when the school food is reserved for the poor the polarisation really affects their morale. »

24. Daily Nation, Nairobi, 30 juillet 2001. 
Mais la décrépitude des établissements du pays a été révélée au monde entier par la tragédie de Kyanguli, en mars 2000, pendant laquelle 67 pensionnaires d'une école secondaire de garçons ont péri ${ }^{25}$. Les conditions d'hygiène et de sécurité ont alourdi le bilan humain de ce drame ; 139 étudiants dormaient dans une chambrée prévue pour 80 et dépourvue d'issue de secours $^{26}$. L'origine criminelle de l'incendie montre la violence qui a gagné les écoles du Kenya. Les attaques, les viols et les incendies se sont, en effet, multipliés au cours de ces dernières années. Ces actes criminels, qui ont désormais l'École pour théâtre, semblent exprimer la frustration, la colère et le désespoir face à une institution scolaire incapable de tenir ses promesses.

Alors que la situation économique du Kenya se dégrade un peu plus chaque année, offrant des perspectives toujours plus réduites aux jeunes diplômés, les responsables politiques enfermés dans des querelles électorales ne parviennent pas à répondre positivement à la crise. Au printemps 2000, le gouvernement s'était pourtant penché sur les problèmes des écoles. Mais les recommandations issues de l'enquête réalisée par M. Koech, et publiées en avril 2000, n'ont pas été validées par le gouvernement. La commission proposait une réforme globale en portant notamment la durée du cycle primaire à 12 ans et en supprimant le $\mathrm{KCPE}^{27}$. Ces dispositions ont été jugées impraticables et trop coûteuses, mais le gouvernement n'a pas proposé de solution alternative, laissant le système éducatif s'enfoncer plus avant dans une crise devenue aujourd'hui ingérable.

Les réponses officielles à la crise apparaissent, en effet, dérisoires. Certains politiciens invoquent le multipartisme ou la magie noire à l'origine de ces problèmes, alors que d'autres, plus pragmatiques, recommandent le rétablissement des châtiments corporels dans les écoles - et notamment la canne qui vient d'être supprimée par le ministre de l'Éducation. C'est une des conclusions à laquelle sont arrivés les directeurs des écoles secondaires réunis à Eldoret, en juin 2001, à l'occasion de leur congrès annuel ${ }^{28}$.

Toute la frustration de la population du Kenya, désemparée par un système scolaire inefficace, semble s'exprimer dans cette crise. L'École ne répond plus aux attentes des Kenyans, puisque les mécanismes de la promotion sociale se trouvent bloqués par une économie déliquescente. Les mesures prises par le gouvernement dans un contexte de libéralisme économique ont, par ailleurs, renforcé les clivages de la société. L'École, qui est devenue le

25. Déjà, le 25 mars 1998, un dortoir de l'école de filles de Bombululu avait explosé. Le gouvernement avait alors nommé une commission d'enquête dont les conclusions n'ont jamais été rendues publiques.

26. Daily Nation, Nairobi, 30 juillet 2001.

27. Daily Nation, Nairobi, 21 août 2000.

28. Daily Nation, Nairobi, 16 juillet 2001. 
lieu d'une vive compétition, ne remplit plus sa fonction sociale d'intégration définie au moment de l'indépendance. La faillite du système scolaire du Kenya est finalement l'expression de la rupture définitive entre les deux logiques qui avaient présidé à son expansion. Le système a, en effet, fonctionné tant que les dynamiques sociales et politiques se complétaient. Mais dans un contexte économique plus difficile, l'éducation ne garantit plus la promotion sociale qui obéit alors à d'autres logiques plus politiques. Mais la déliquescence rapide de l'institution scolaire du Kenya met en péril les formidables progrès réalisés depuis l'indépendance. L'éducation, loin de renforcer la cohésion de la société, accentue les différenciations sociales dans une société de plus en plus clivée. L'ampleur et la violence de la crise qui secoue aujourd'hui les écoles du Kenya sont à la hauteur des espoirs déçus.

Université Paris 7 - Denis Diderot, Paris.

\section{BIBLIOGRAPHIE}

ABREU, E.

1975 The Role of Self Help in the Development of Education in Kenya 1900-1973, Nairobi, Kenya Literature Bureau.

Aduda, D.

2001 «Pathetic Conditions in Kenya's Boarding Schools Revealed», Africa on Line, 30/04/2001.

BANQue Mondiale

1988 Éducation en Afrique subsaharienne. Pour une stratégie d'ajustement, de revitalisation et d'expansion, Washington, World Bank.

BogONKO, S.

1992 A History of Modern Education in Kenya (1895-1991), Nairobi, Evans Brothers.

Charton, H.

1997 Le désir d'école, Paris, Publications de l'Université Paris 7-Denis Diderot.

EshiwANI, G. S.

1993 Education in Kenya Since Independence, Nairobi, East African Educational Publishers.

Hazlewood, A.

1989 Education Work and Pay in East Africa, Oxford, Clarendon Press. 
KenyatTA, J.

1965 Harambee! The Prime Minister of Kenya's Speeches: 1963-64, from the Attainment of Internal Self-government to the Threshold of the Kenya Republic, Nairobi, Oxford University Press.

Kinyanjui, P.

1971 «Education Training and Employment of Secondary School Leavers », Education in Eastern Africa, $2: 3-21$.

LEWINGER MoOcK, J.

1973 « Pragmatism and the Primary School, the Case of a Rural Village », Africa, $43: 302-316$.

Mbeo Manyasi

2000 «Working to Restore Past Glories », International Labor Office, Africa Newsletter, 2.

Ogot, B. A. (ed.)

1995 Decolonisation and Independence in Kenya, Nairobi, East African Studies.

SifunA, D.

1980 Short Essays on Education in Kenya, Nairobi, East African Literature Bureau.

\section{RÉSUMÉ}

Le taux de scolarisation primaire du Kenya - longtemps pays modèle en matière d'éducation —, est passé de $95 \%$ en 1988 à $79 \%$ en 2000. Ce phénomène de déscolarisation qui se double de violences et de grèves inédites, nous invite à nous interroger sur les facteurs historiques qui ont précipité le déclin de l'institution scolaire du Kenya. À l'époque coloniale, la soif d'éducation des populations africaines s'est exprimée à travers des formes originales de mobilisation populaire. À l'indépendance du Kenya en 1963, l'École a joué un rôle fédérateur et structurant dans l'émergence de la nation kenyane. Les initiatives populaires ont, en effet, été encouragées et institutionnalisées par les responsables de la jeune république qui a alors connu une scolarisation rapide et massive. Mais depuis dix ans, les difficultés économiques du pays et le désengagement manifeste du pouvoir politique ont rompu cette dynamique. La société civile a perdu confiance dans un système scolaire ruineux et corrompu. La violence de la crise que traversent aujourd'hui les institutions scolaires du Kenya est alors à l'image de l'ampleur de la déception d'une population qui s'est beaucoup investie dans l'éducation de ses enfants.

\section{ABSTRACT}

The Collapse of the Kenyan Educational System, Points for a Historical Analysis. For a long time, Kenya was a model as regards education; but the enrollment rate in primary schools fell from $95 \%$ in 1988 to $79 \%$ in 2000 . This drop has come along with violence and unprecedented strikes. What historical factors have caused 
the Kenyan educational system to collapse? During the colonial era, Africans longed for education, and this spawned original forms of mass mobilization. At the time of independence in 1963, schools played a unifying role that shaped the new nation. The young republic's leaders encouraged and institutionalized grassroots initiatives; and school enrollment rates shot up. During the past ten years, this momentum has been thwarted by the country's economic problems and the government's withdrawal of support. People have lost confidence in a corrupt, ruinous school system. The violence in the school crisis reflects the intense disappointment felt by a population that invested in educating its children.

Mots-clés/Keywords : Kenya, déscolarisation, écoles privées, éducation, élitisme, grèves scolaires, initiatives populaires, libéralisme économique, violences scolaires/ Kenya, education, elitism, economic liberalism, private schools, grassroots initiatives, school violence, school strikes. 